\title{
Spinal excision osteotomy in myelomeningocele: with special reference to internal fixation
}

\author{
GeOFFREY WALKER \\ F.R.C.S.
}

\author{
Philip Cheong-Leen \\ F.R.C.S.
}

\section{Queen Mary's Hospital for Children, Carshalton, Surrey}

THE aetiology and pathology of progressive lumbar kyphosis in children born with myelomeningocele have been well described by Sharrard and Drennan (1972). In this paper we describe our present technique for correcting this severe deformity, and we discuss the results obtained in eighteen Spina Bifida children between 2 and 11 years of age.

\section{Material and indications}

We have performed twenty operations in eighteen children during the last 6 years. The indications were recurrent skin ulceration in fourteen, and a bizarre and gross deformity in four (Fig. 1). None of the

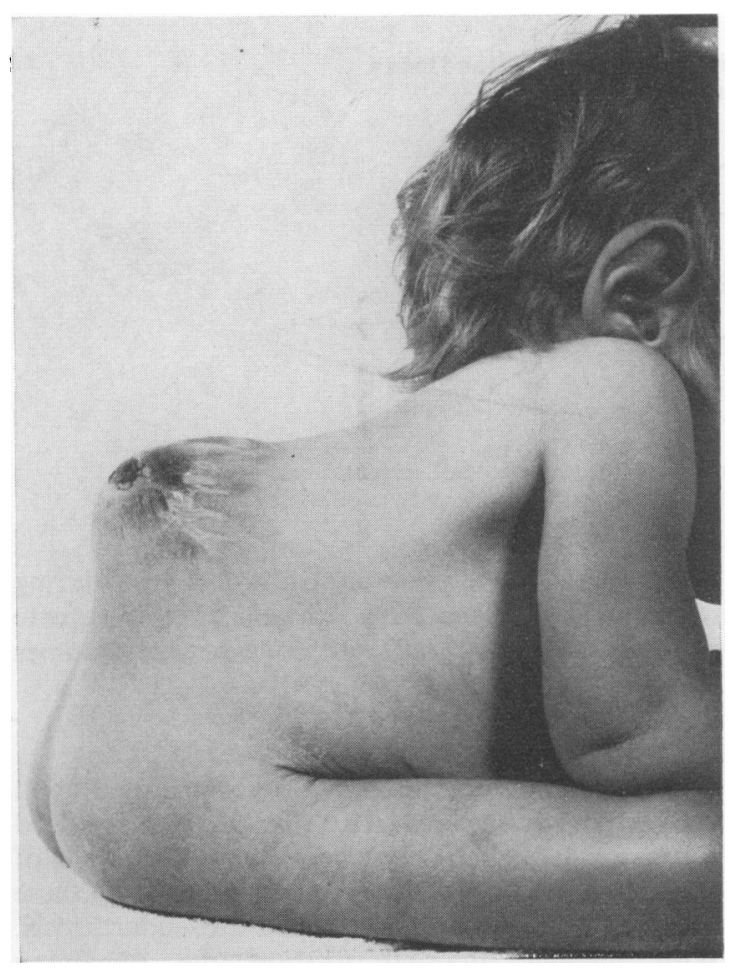

FIG. 1. Recurrent skin ulceration is a major problem over these angular kyphoses. children had any useful muscle power in either lower limb. Two repeat operations have been performed as the deformity recurred (vide infra). There were thirteen boys and five girls, and the majority were aged between 2 and 7 years at the time of surgery. The results of psychological testing were available in fourteen, and ten were in the 'normal' range although dull (albeit in the lower grades).

\section{Operation}

Under general anaesthesia (the assistance of a skilled and experienced anaesthetist is essential), and with the child prone an incision is made to allow exposure of the dura and vertebrae. In our experience it has been safer to re-open the previous vertical incision rather than to convert this to a cruciate wound by routinely using the transverse incision advocated by Sharrard and Drennan (1972); as the skin in this area has a poor blood supply and the four apices of a cruciate scar are prone to necrosis. Decision as to the placement of the incision must, however, always depend on the extent of previous surgery, and we are fortunate at Queen Mary's Hospital for Children, as primary closure is usually completed with a simple vertical scar, uncomplicated by flap or transverse incisions. The dura is mobilized and retained, and this allows exposure of the posterior aspect of the spinal column (Fig. 2). Through small incisions made just along side the lateral masses, a suitable instrument, and then a finger is insinuated anterior to the vertebral body at the apex of the deformity (Fig. 3). This exposure is extended proximally and distally by blunt dissection until adequate exposure is obtained. With the dura retracted osteotomies are then performed and it is usual to remove one and two half vertebral bodies (Fig. 4). The angles of the osteotomies can best be gauged at operation, and are designed to allow maximum correction with good apposition of the cancellous bone surfaces of the vertebral half bodies which remain. Fixation is important, and we now use two double wires (SWG 29) passed through the conveniently situated intervertebral foraminae on either side of the osteotomy (Fig. 5a, and b). In 

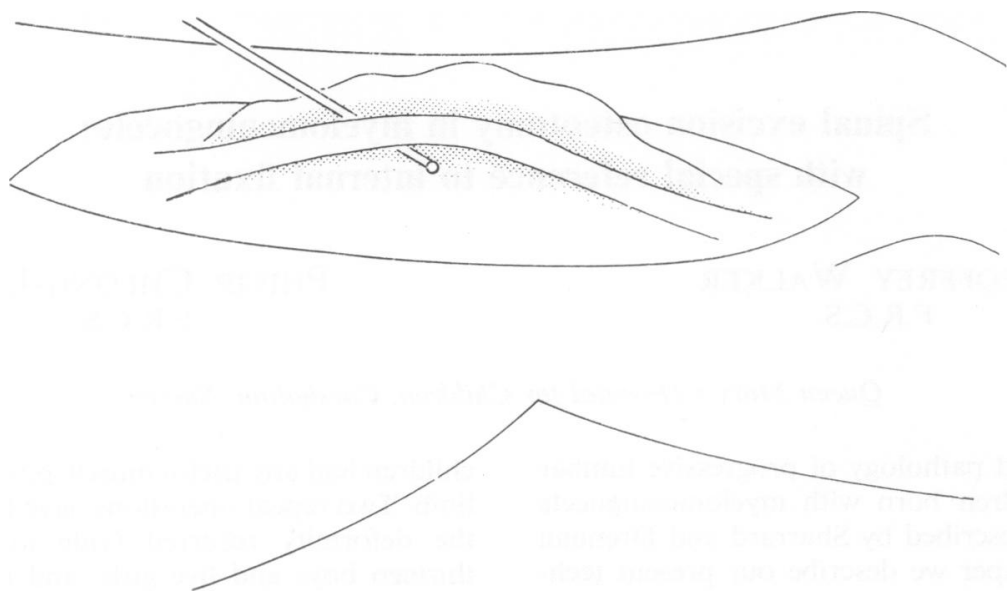

FIG. 2. The dura is mobilized and retained.

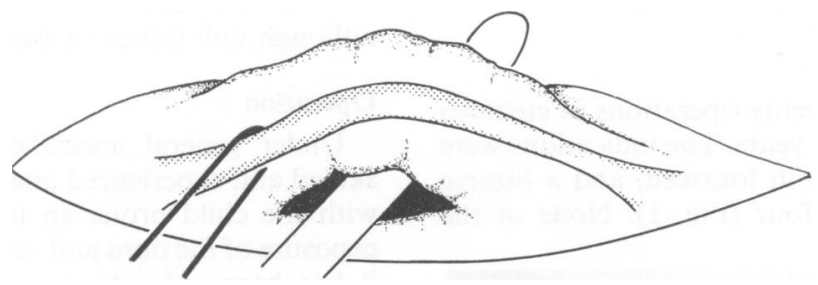

Fig. 3. Through two small lateral incisions, an instrument and then a finger is passed anterior to the vertebral column.

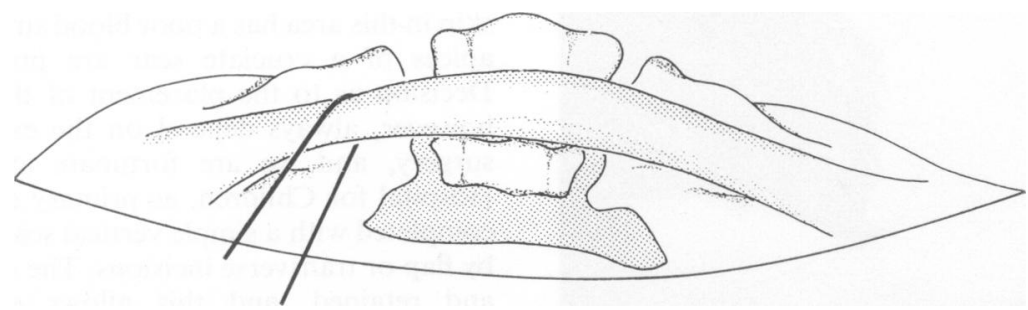

FIG. 4. Osteotomies are performed to allow removal of one and two half vertebral bodies with apposition of the remaining contiguous surfaces, and as much correction as possible.

addition one or two AO lag screws are used to further compress the osteotomy (Fig. 6a, b and c). These may be inserted either proximally or distally, and although their use means that total correction cannot have been achieved, the firm fixation they produce is well worthwhile. The dura will be found to cover the heads of the screws and the osteotomy, and should be lightly sutured in place, it provides good subcutaneous tissue between the skin and the spinal column (Fig. 7). The skin is carefully closed with interrupted sutures over a suction drain. With this type of rigid fixation, it has been possible to dispense with plaster splintage, and the children are nursed free in bed being turned at regular intervals for 3 weeks. The amount of blood lost during operation has been very variable, but adequateo replacement is essential, and considerable volumes may have to be transfused.

\section{Results}

Of the eighteen children, two were operated upon over 6 years ago using a strut of 'Kiel' bone wedged across the defect after excision osteotomy. In spite of immobilization in plaster for 6 months, both of theses? failed to fuse and each developed a pseudarthrosis. 0 With metallic internal fixation the remainingo eighteen, which have all been performed in the last命 3 years, have rapidly united. However, it is only sincep 

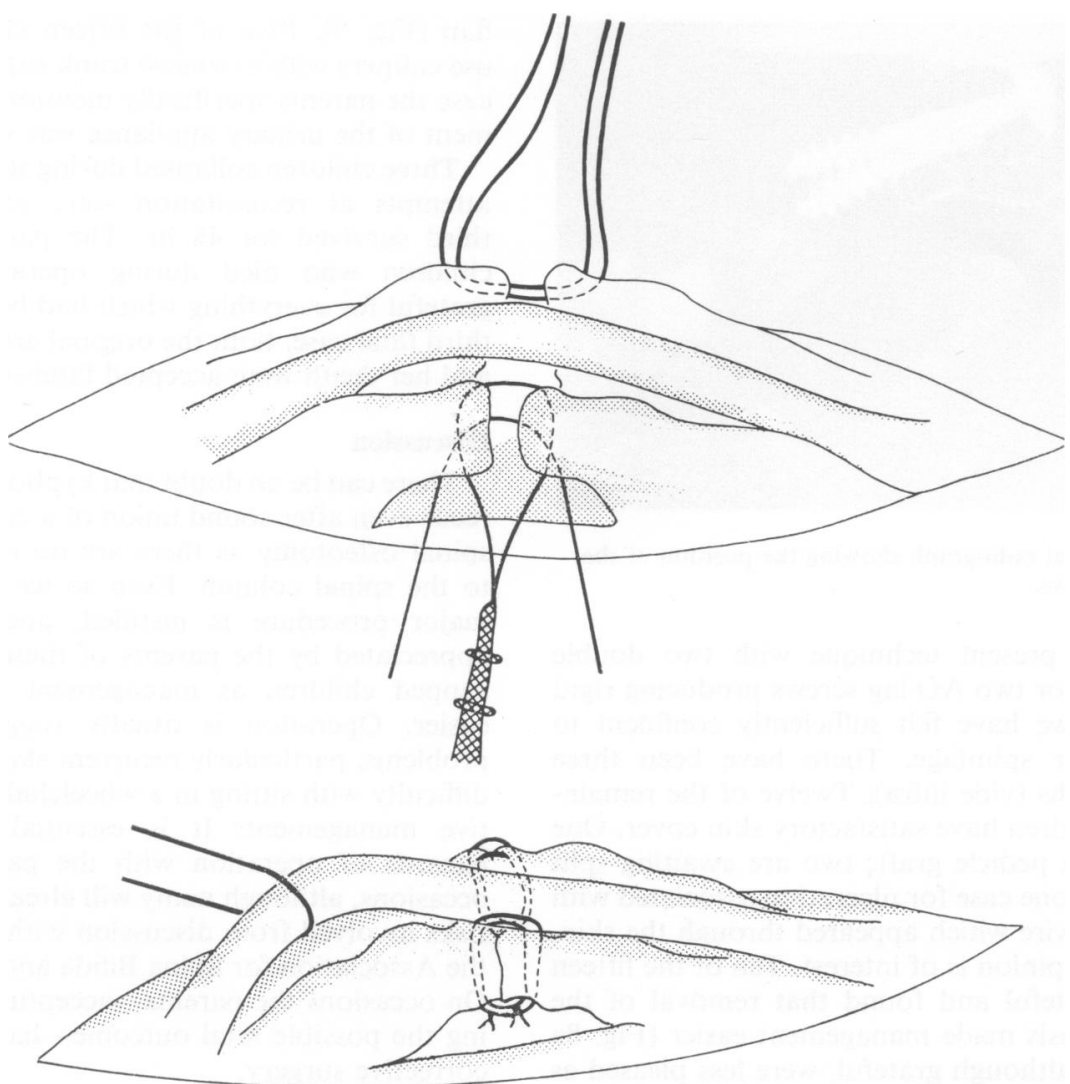

Fig. 5. (a \& b) Double wires are passed through the intervertebral foraminae and tightened.
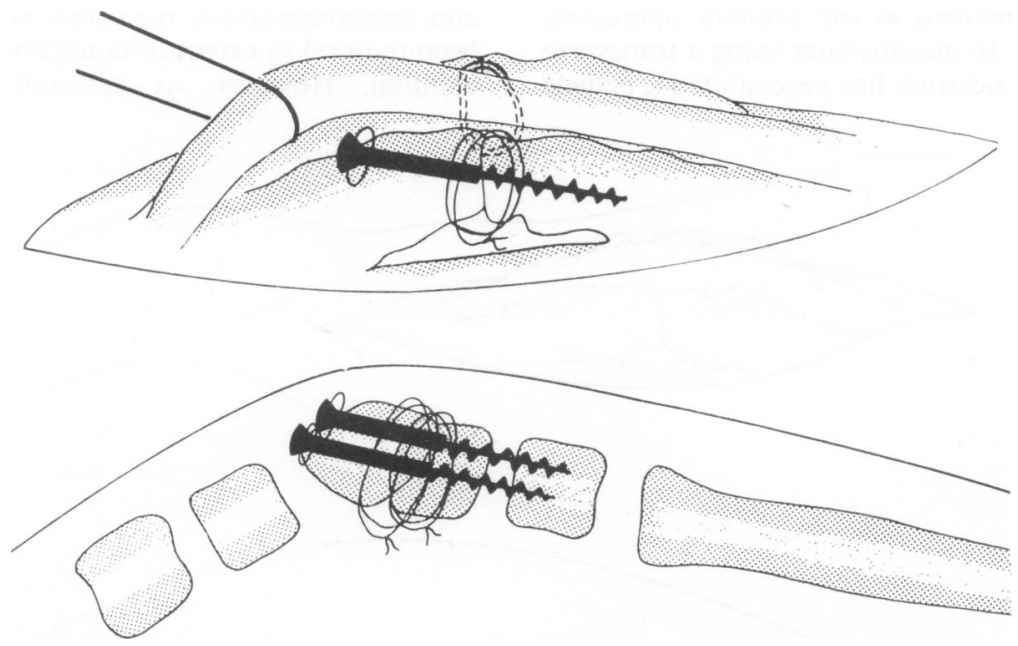

Fig. 6. (a \& b) AO lag screws are used to further compress the osteotomy site. 


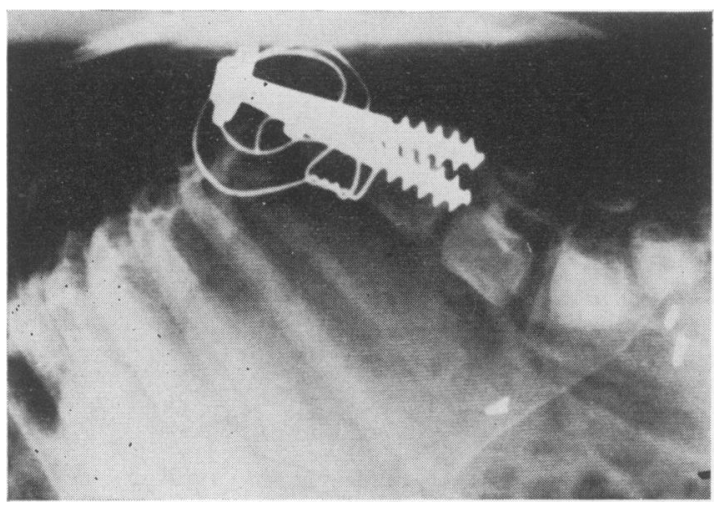

Fig.6(c) Lateral radiograph showing the position of the wires and screws.

adopting our present technique with two double wires and one or two AO lag screws producing rigid fixation that we have felt sufficiently confident to discard plaster splintage. There have been three operative deaths (vide infra). Twelve of the remaining fifteen children have satisfactory skin cover. One has required a pedicle graft; two are awaiting split skin grafts, in one case for ulceration associated with removal of a wire which appeared through the skin. The parents' opinion is of interest. Ten of the fifteen were very grateful and found that removal of the angular kyphosis made management easier (Fig. 8a and b). Five, although grateful, were less pleased as either there was early recurrence of the deformity or persistent minor skin ulceration. Two repeat operations have so far been required. One in a child who developed a pseudarthrosis with recurrence of deformity 6 years after an abortive Kiel bone graft. In the other, an older boy of 11 years, only moderate correction was obtained at the primary operation. This was repeated 16 months later using a transverse incision, and skin necrosis has necessitated a pedicle flap (Fig. 9). Five of the fifteen children regularly use calipers with extensive trunk support, and in one case the parents specifically mentioned that management of the urinary appliance was simplified.

Three children collapsed during surgery and in two attempts at resuscitation were unsuccessful. The third survived for $48 \mathrm{hr}$. The parents of the two children who died during operation were 'very grateful for everything which had been done'. In the third fatal case, both the original arrival of the child, and her death were accepted fatalistically.

\section{Discussion}

There can be no doubt that kyphosis will inevitably recur even after sound union of a corrective excision spinal osteotomy as there are no muscles posterior to the spinal column. Even so we believe that this major procedure is justified, and it is certainly appreciated by the parents of these severely handicapped children as management is made a little easier. Operation is usually suggested when the problems, particularly recurrent skin ulceration, and difficulty with sitting in a wheelchair, defy conservative management. It is essential to discuss the hazards of operation with the parents on several occasions, although many will already appreciate the risks involved from discussion with other parents in the Association for Spina Bifida and Hydrocephalus. On occasions the parents-accepting and appreciating the possible fatal outcome-have suggested this corrective surgery.

Our first attempts at metallic internal fixation were with intervertebral wiring, but this necessitated prolonged plaster fixation. The addition of Shermann bone screws, later superseded by AO lag screws, allows us to discard plaster, and the freedom which results is appreciated by the children, nursing staff and physiotherapists. Recurrent skin ulceration has been reduced in extent or eradicated in nearly all the children. However, as deformity inexorably re-

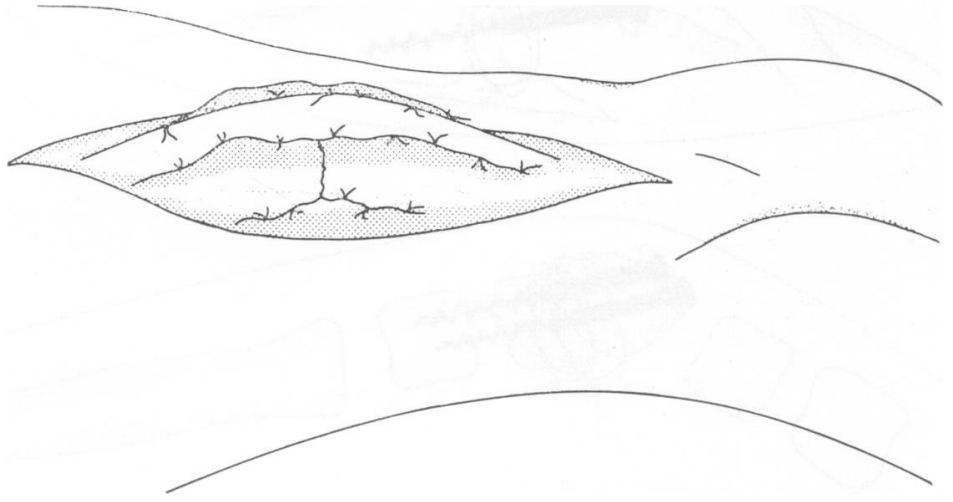

FIG. 7. The dura is replaced and covers the wires and screws. 


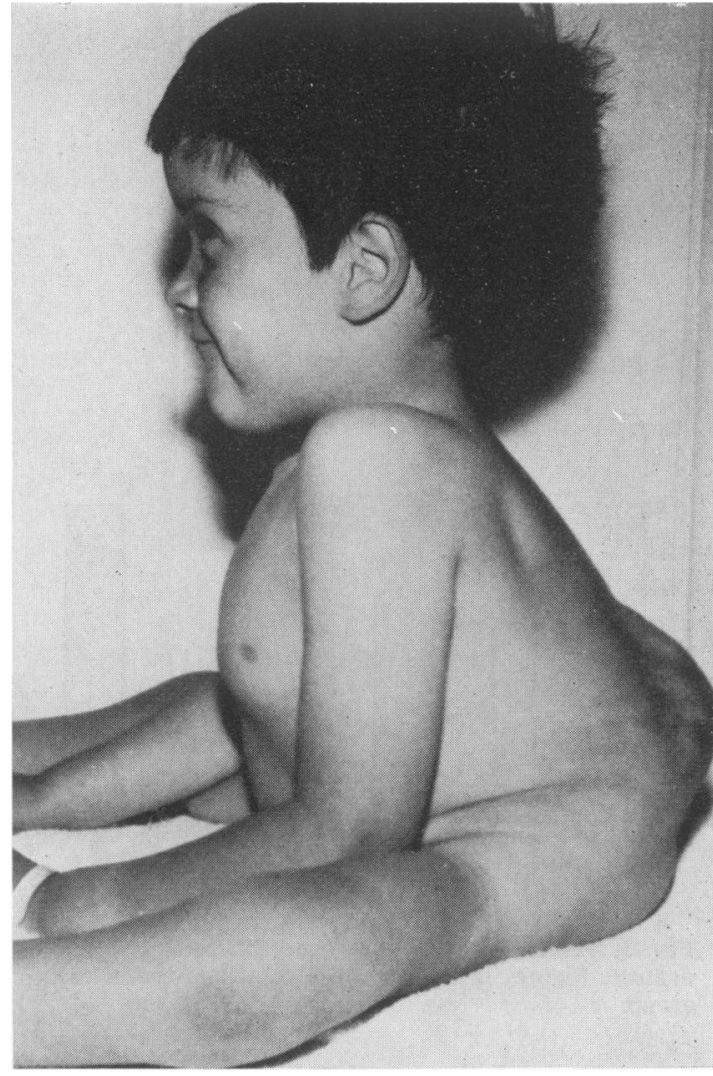

Fig. 8. (a \& b) Pre- and post-operative photographs to show the amount of correction which can be obtained

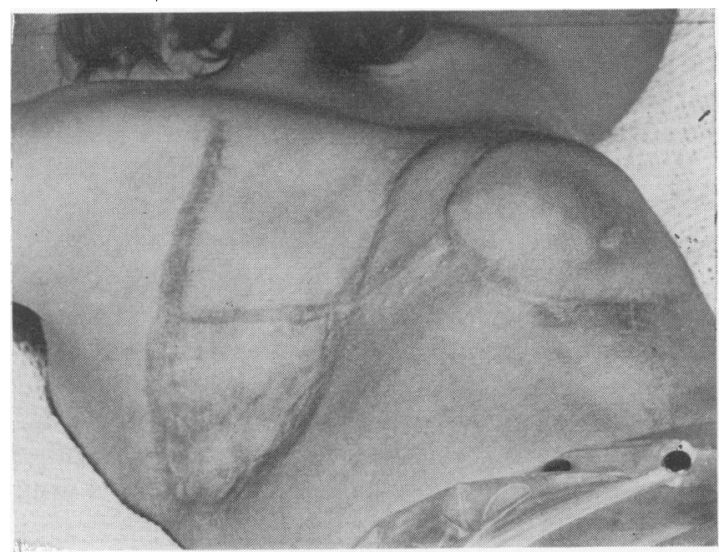

FIG. 9. A pedicle flap was required in this boy after two osteotomies, the second with a 'cruciate' scar.

appears, importation of full thickness skin and repeat bone operations will become inevitable in some children.

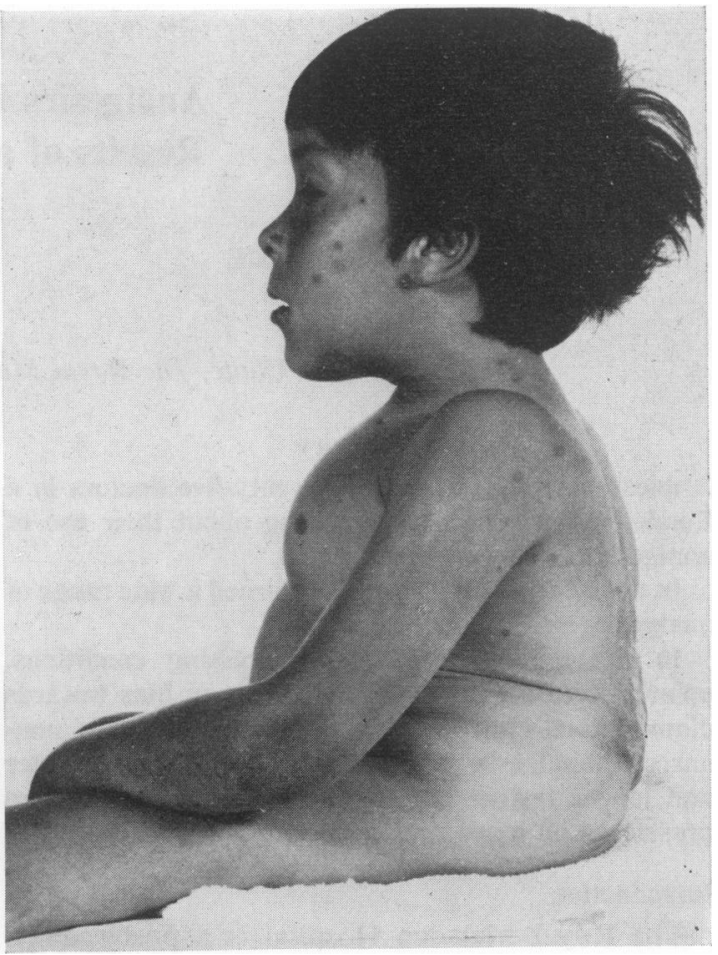

Figure 8b.

While there is no doubt that excision spinal osteotomy can help these severely handicapped children and their parents, it is to be hoped that if the teaching of Dr John Lorber (1971) becomes widely accepted, it will be these paralysed, incontinent, and deformed children needing this extensive surgery, who will be 'selected out' during the first weeks or months of life.

\section{Acknowledgments}

Our thanks are due to Miss Mary Pugh, Miss P. J. Philpott and $\mathrm{Mr}$ N. Le Page for the illustrations. Mrs Brenda Beal, M.S.C.P., kindly prepared the diagrams, and Miss Susan Wadd typed the manuscript.

Four of the children were operated on by G.W. in association with Mr John Wilkinson and Mr Richard Howard, and we are grateful for their co-operation.

\section{References}

LORBER, J. (1971) Results of treatment of myelomeningocele. An analysis of 524 unselected cases, with special reference to possible selection for treatment. Developmental Medical Child Neurology, 13, 279.

SharRaRd, W.J.W. \& DrennaN, J.C. (1972) Osteotomyexcision of the spine for lumbar kyphosis in older children with myelomeningocele. Journal of Bone and Joint Surgery, 54B, 50. 